\title{
Review
}

\section{Advances in Studies on Hepatitis E Virus}

\author{
Weiying Zhang \\ College of Life Sciences, Nankai University, Tianjin China
}

\author{
Keywords \\ Hepatitis E, Hepatitis E virus, Epidemiology

\section{Correspondence} \\ Weiying Zhang, \\ E-mail: wyzhangnk@126.com
}

DOI: 10.1515/ii-2017-0117

\begin{abstract}
Hepatitis E virus (HEV) is transmitted mainly through food and water sources. HEV is a major public health problem faced by developing countries. HEV causes large outbreaks of hepatitis $\mathrm{E}$ (HE) or acute sporadic infection. HEV can also lead to chronic infection. HEV mainly affects the young, middle-aged people, and pregnant women, who especially display high rates of infection. This study reviews HE while considering its biological characteristics, clinical symptoms, epidemic characteristics, vaccine, and detection methods.
\end{abstract}

Hepatitis E (HE) is an acute epidemic hepatitis caused by $\mathrm{HE}$ virus ( $\mathrm{HEV})$, which propagates mainly through fecal-oral channels. HE is also called non-a, non-b hepatitis and infects by enteric transmission. This study briefly reviews advances in studies of HEV.

\section{Biological Characteristics}

In 1983, using immunoelectron microscopy, Balagan et al. observed 27-30 nm diameter virus-like particles in feces of a volunteer patient and proved that this organism was a new non-a, non-b hepatitis virus. In 1989, the Tokyo International Conference officially named this virus as hepatitis E virus (HEV).

$\mathrm{HEV}$ is a non-enveloped positive-sense single-stranded RNA virus. Overall length of the genome measures $7.5 \mathrm{~kb}$ with three open reading frames (ORF) (i.e., ORF1, ORF2, and ORF3), $5^{\prime}$ end noncoding regions, and $3^{\prime}$ end noncoding regions. ORF1 is the largest, contains 1693 codons, and encodes methyltransferase, helicase, RNA-dependent RNA polymerase, and nonstructural protein of papain-like serine protease. Hypermutation region in ORF1 may be associated with pathogenicity of the virus. ORF2-encoding envelope protein contains a plasmid recovery signal protein and 3 ' $\mathrm{N}$-glycoprotein locus. ORF3 is small and plays an important role in virus replication and aggregation.

In 1989, Reyes et al. applied molecular cloning technique to obtain HEV gene clone. By sequencing its entire genome, $\mathrm{HEV}$ is divided into four genotypes. HEV I causes acute infection outbreaks in Asian population. HEV II results in outbreaks in some American populations. HEV III infection mainly occurs in industrialized countries, mainly as crowd distribution and chronic infection and infection of pigs, deer, mongoose, rats, and rabbits. HEV IV infection is also endemic, mainly causing distributed infection in crowds and pigs, cattle, and sheep. HEV I and IV genotypes were identified in China, with HEV IV showing dominance with characteristics of regional distribution and presenting different subtypes. From 2010 to 2012, studies performed in Liaoning and Hebei provinces indicated that $90.3 \%$ of isolates comprised HEV IV type with $4 \mathrm{a}, 4 \mathrm{~b}, 4 \mathrm{~h}, 4 \mathrm{i}$, and another new gene subtype. One isolate was HEV IIIa type, and another three isolates were HEV Ib type ${ }^{[1]}$. Based on genotype analysis of HEV strains collected in Shanghai from 1997 to 2012, $3979 \mathrm{HEV}$ strains were type IV and were divided into $4 \mathrm{a}, 4 \mathrm{~d}, 4 \mathrm{~h}$, and $4 \mathrm{I}$ subtypes ${ }^{[2]}$.

\section{Clinical Symptoms}

HE is generally inspected and diagnosed based on 
epidemiology data, symptom, signs, and experimental examination. After $\mathrm{HEV}$ infection, incubation period lasts for three to eight weeks. The most infectious period is the end of incubation period and early stage of acute period. Subsequently, patients usually exhibit viremia. The liver is the primary organ of HEV infection, and the virus is discharged into bile duct until it reaches high concentrations and is then excreted by stools. Viremia and fecal detoxification precede liver abnormalities. HEV was isolated from cerebrospinal fluid of patients with chronic infection. Therefore, sites of extrahepatic replication of HEV still require further studies. Clinical symptoms usually include fatigue with three- to seven-day nausea, epigastric discomfort in the right upper quadrant, and dark urine and jaundice. Severity of disease varies with people. HEV infection usually manifests as acute self-limited disease with normal recovery period of two to eight weeks. However, in developed countries, HEV can cause chronic infection of organ transplant patients, leading to rapid progress of liver cirrhosis. HEV also results in extrahepatic clinical symptoms, such as nervous system symptoms and kidney damage. In general, acute infection does not require treatment, but transplant patients and/or patients using antiviral therapy should reduce usage of immunosuppressive agents for chronic infection ${ }^{[3]}$. Outbreak of $\mathrm{HE}$ in pregnant women, especially in their late pregnancy, easily leads to high morbidity, high mortality, high acute liver failure, and liver disease and premature infant birth. Thus, risk of low birth-weight infants and perinatal mortality increases. Causes of severe HEV symptoms in pregnant women must still be clarified though they may be genotypes of HEV and other pathogens. Studying the mechanism of severe $\mathrm{HEV}$ infection associated with pregnancy will provide effective measures to prevent HE.

\section{Epidemic Characteristics}

HEV is globally distributed and is one of the most important pathogens of acute viral hepatitis in most developing countries. Ning et al. ${ }^{[4]}$ analyzed HE epidemic characteristics from 2004 to 2011 in China and observed increasing trend in $\mathrm{HE}$ incidence and constituent ratios in five kinds of viral hepatitis. The five highest average morbidities were respectively observed in Liaoning, Jiangsu, Zhejiang, Shanghai, and Tianjin. In 2012, 28,172 newly infected HE patients were observed in China ${ }^{[5]}$. Using time series decomposition and circular distribution, Shan et al. ${ }^{[6]}$ analyzed seasonal variation factors of $\mathrm{HE}$ cases in China from 2005 to 2010. These researchers discovered that peak times of HE were winter and spring, especially at the end of March and beginning of April. These findings agreed with results obtained by Ning et al. ${ }^{[4]}$, who showed that peak period lasted from January to May. HEV transmission routes mainly include transmissions through water sources, food, contact, and maternal and infant vertical transmission. Xuexia et al. ${ }^{[7]}$ observed that HEV infection in Guangzhou showed familial aggregation and ease of coinfection of HEV by daily contact. Therefore, we should strengthen family-based prevention and control awareness. HEV can cause large-scale outbreaks, acute sporadic HE, and chronic infection. Development of chronic infection in immunocompromised patients after HEV infection represents an urgent and important clinical issue. Thus, studies should determine the relationship between host immunity and other factors leading to chronic infection. In addition to humans, HEV also infects animals, such as pigs, deer, chicken, rats, fish, rabbits, and ferrets. Therefore, food safety related to HEV pollution should also increase wide-range public safety awareness ${ }^{[8]}$.

\section{Vaccine}

Cell culture systems are only confined to types HEV III and IV, and infectious cDNA clones were successfully prepared. Okamoto et al. used human lung cell A549 and human hepatocytes PLC/prf/5 to successfully culture HEV III and IV. Emerson et al. successfully cultured HEV III by using human liver cell line HepG2/3A. Success of cell culture systems benefited in vitro studies of inactivated and attenuated vaccines ${ }^{[9]}$.

To date, genetic engineering vaccines mainly use prokaryotes, yeast, and plant cell systems for expression products of HEV ORF2 protein. These vaccines are divided into recombinant proteins, nucleic acids, HEV particle-like vaccine, and transgenic plant vaccines. Based on HEVE2, HEV239 (Hecolin) vaccine developed using prokaryotic cells in China passed phase I/II/III clinical trials from 2004 to 2009 . This vaccine showed good results in three aspects, namely, antigenicity, immunogenicity, and safety ${ }^{[10]}$. The vaccine was listed in China in 2012. However, HEV 239 only prevents infection and diseases caused by HEV genotypes I and IV. Further clinical trial validation is still needed regarding effectiveness of HEV 239 to genotypes II and III and other high-risk groups, such as pregnant woman and 
hepatic disease patients.

At the fundamental research and clinical trial stage, $\mathrm{HEV}$ vaccine considers humoral immunity an assessment index. Cellular immunity also plays an important role in resistance to HEV. Meng et al. constructed target cells of HEV based on p179 segment of HEV ORF2 C and discovered that cell killing rate of 179 protein was significantly lower than that of recombinant plasmid. Possibly, gene immunity easily induced cellular immunity ${ }^{[11]}$.

\section{Detection Method}

Methods were established for HEV IgG antibody, IgM antibody, antigen, and nucleic acid biomarkers, providing possibilities to analyze the relationship between various markers of HEV and effects of early HE diagnosis.

\section{Virology Detection of HEV}

After oral infection, the intestine may serve as primary replication site. Then, infection may spread to the liver. HEV is replicated in liver cell cytoplasm, released into bile and blood stream, and then drained into the intestinal tract. In general, the virus can be detected from blood and feces one to three weeks after infection (approximately one week before onset). During incubation period and early acute stage of $\mathrm{HE}$, immune technique presents high detection rate in stool or bile.

\section{Serum Immunology Detection}

Specific IgM antibody and IgG antibody can be detected in sera two to four weeks after infection. Anti-HEVIgA can be used as supplement to $\mathrm{HEV}-\mathrm{Ig} M$ in recent infection. Combined detection of HEV-IgA and IgM can improve diagnostic sensitivity of acute $\mathrm{HE}{ }^{[12]}$. In late acute phase, IgM antibody regresses rapidly, and IgG antibody persists in the long term. Anti-HEV-IgG positivity cannot be used as criterion because of the difficulty posed by distinguishing previous and acute infections. Anti HEV - IgG response occurs shortly after IgM reaction and increases continuously in the acute phase until recovery period. To date, clinical trials of HE diagnosis are based on enzyme-linked immunosorbent assay (ELISA). However, existing commercial HE ELISA diagnostic kits include different envelope antigens. Differences in genotype are not considered in the design and selection of antigen proteins; thus, diagnostic kits from different companies detection yield significant differences, inadequate sensitivity, and high omission factor $^{[13]}$. Conformational epitope antigens of $\mathrm{HEV}$ include ORF2.1 and NE2 antigens expressed by Escherichia coli and $55 \mathrm{kD}$ antigen expressed by insect baculovirus systems. The new HEV diagnostic reagent established based on this information exhibits high sensitivity and specificity [14].

\section{Serum Nucleic Acid Detection}

In patients with $\mathrm{HEV}$ infection, during the window period or due to influence of autoimmune status, serum anti-HEV antibody may not be produced or fail to reach detection level, resulting in failure of detection by serological methods. HEV RNA is translated during early stage of $\mathrm{HEV}$ infection, staying for short periods in the blood. Polymerase chain reaction (PCR) is an important tool for diagnosis of HEV RNA in sera and/or feces. Detection of HEV RNA at the early stage of infection can compromise deficiencies in serological detection, exhibiting important clinical significance for early diagnosis of HE. However, viremia primarily causes early-stage acute $\mathrm{HEV}$ infection, and PCR negative results do not rule out the possibility of HEV infection. PCR is mainly used to monitor progress of chronic HEV infection. No unified standard is available for molecular diagnosis of $\mathrm{HEV}$ and monitoring in different laboratories and countries.

Regional distribution of HEV genotypes, constant discovery of animal hosts, and lack of gold standard for laboratory serological diagnosis increased difficulties in prevention and control of HEV infection. In short, effective measures for controlling $\mathrm{HEV}$ infection should include improving personal and public health, strengthening food safety awareness, and developing $\mathrm{HEV}$ vaccine research for high-risk groups, especially pregnant women.

\section{Declarations}

\section{Acknowledgements}

No.

\section{Competing interests}

The author declares that she has no competing interest.

\section{Authors' contributions}

WY Zhang made the literature analysis and wrote, discussed and revised the manuscript of this review. 


\section{References}

1 GENG YS, ZHAO CY, FAN JP, et al. Genotype analysis of hepatitis E virus from sporadic hepatitis E cases in northern China. Infect Genet Evol, 2013, 20:413-417.

2 REN H, LI YT, ZHOU X, et al. Epidemiologic and genetic characteristics of hepatitis E virus in Shanghai, 1997-2012. Zhonghua Liu Xing Bing Xue Za Zhi, 2013,34 (5):419-423.

3 Kamar N, Dalton HR, Abravanel F, et al. Hepatitis E virus infection. Clin Microbiol Rev. 2014 Jan;27 (1):116-138.

4 Miao Ning, Zhang Guomin, Gong Xiaohong, et al. The Epidemiological Analysis of Hepatitis E in 2004-2011 in China. Chinese Journal of Vaccines and Immunization, 2013,19 (5):451-454.

5 Zhang L, Wang Y, Chen Y, et al. Review of Infection Disease in 2012. Infectious Disease Information, 2013, 26 (1):10-16.

6 Wei S, Lu Y, Gao M, et al. Time Series Analysis of Hepatitis E Incidence in China. Chinese Journal of Health Statistics, 2012,29 (6):808-811.

7 Yun X, Liu J, Cai Y, et al. Family Aggregation Characteristics Analysis of Hepatitis A, Hepatitis B and Hepatitis E Virus in Guangzhou. Modern Preventive Medicine,2011,38 (19):3877-3880.

8 Yugo DM, MENG XJ. Hepatitis E virus: foodborne, waterborne and zoonotic transmission. Int J Environ Res Public Health, 2013,10 (10):4507-4533

9 Suzanne E. Molecular Virology of HEV [C] //An NIH Research Workshop. Hepatitis E in the United States. Bethesda, MD: National Institutes of Health,2012.

10 ZHU FC, ZHANG J, ZHANG XF, et al. Efficacy and safety of a recombinant hepatitis $\mathrm{E}$ vaccine in healthy adults: a large-scale, randomised, double-blind placebo-controlled, phase 3 trial. Lancet, 2010, 376(9744):895-902.

11 Yang S, Wang C, Wu Y, et al. Construction and Application of Target Cells Based on HEV-p179 of Neutralizing Epitope of Hepatitis E Virus. Chinese Journal of Clinical Laboratory Science, 2012, 30 (8):599-601.

12 Zhang L, Pan J, Zhou J, et al. Evaluation of Specific IgA against Hepatitis E Virus (HEV) in the Diagnosis of Acute HEV Infection.Chinese Journal of Health Laboratory Technology, 2010, 20 (8):1951-1953.

$13 \mathrm{Xu} \mathrm{Q}$, Wang W. Early Identification and Control for Hepatitis C in General Hospital. Chinese Journal of Nosocomiology, 2011, 21 (17):3573-3575.

14 Liu Z, Wen W, Li D. Research Progress in Laboratory Diagnostic Methods for HEV Infection. Modern Preventive Medicine, 2011, 38 (19):3999-4000. 